\title{
Synthesis and Properties of Linear Polyether-Blocked Amino Silicone-Modified Cationic Waterborne Polyurethane
}

\author{
Chengshu Xu $\mathbb{D}^{\mathbb{1}},{ }^{1}$ Lei OuYang, ${ }^{2}$ Yan Ren, ${ }^{1}$ Zaisheng Cai, ${ }^{3}$ Shaofeng Lu, ${ }^{1}$ Jing Zhang, \\ and Wenzhao Shi ${ }^{1}$ \\ ${ }^{1}$ School of Textile Science and Engineering, Xi'an Polytechnic University, Xi'an 710048, China \\ ${ }^{2} X i$ 'an Wanzi Fine Chemical Technology Co., Ltd., China \\ ${ }^{3}$ College of Chemistry, Chemical Engineering and Biotechnology, DongHua University, China
}

Correspondence should be addressed to Chengshu Xu; xcs7910@163.com

Received 30 October 2019; Revised 22 January 2020; Accepted 5 February 2020; Published 8 April 2020

Academic Editor: Anjanapura V. Raghu

Copyright (c) 2020 Chengshu Xu et al. This is an open access article distributed under the Creative Commons Attribution License, which permits unrestricted use, distribution, and reproduction in any medium, provided the original work is properly cited.

\begin{abstract}
In this study, a waterborne polyurethane (WPU) is synthesized by using polytetramethylene ether glycol (PTMEG) to form the soft segment, 1,4-butanediol (BDO) as the chain extender, n-methyldiethanolamine (MDEA) as a hydrophilic chain extender, and isophorone diisocyanate (IPDI) to form the hard segment. Furthermore, the modified cationic WPU emulsion and its films are created through a reaction between the WPU and a linear polyether-blocked amino silicone (LEPS), which is an organosilicon compound that imparts flexibility. The properties of the structure and formed WPU films are then characterized by using Fourier transform infrared spectrometry, a thermogravimetric analysis, atomic force microscopy, X-ray diffraction, and X-ray photoelectron spectroscopy, as well as by measuring the water contact angle, testing the water absorption, etc. It is found that, with an increase in the LEPS content in the WPU, the particle size of the modified WPU emulsion is increased, the WPU films are more flexible, and the resistance of the modified WPU films to heat and water are increased, while the crystallinity is reduced. The polysiloxane chain segment, which is added to the LEPS-modified WPU emulsion, is significantly enriched on the surface of the modified WPU films, while there are no adverse effects of the LEPS-modified WPU emulsion on the adhesive force between the WPU and substrate. When the LEPS content of the WPU is $14.0 \mathrm{wt} \%$, the modified WPU emulsion and film provide the best performance.
\end{abstract}

\section{Introduction}

Waterborne polyurethane (WPU), as a functional environmental protection material, is widely used for coating floors and walls in construction sites, in finishings for furniture, coating of leather, 3D printing, fixing reactive dyes, bonding interior materials in automobiles, and binding ink, as a biomaterial for cardiovascular applications in medicine, and in a variety of other different industries [1-6]. However, compared to solvent-based polyurethane (PU), coatings that use WPU have low resistance to water, solvents, heat, and different climates. Therefore, research work on the modification of WPU to produce a WPU with better properties has become very popular.

WPU that is modified with polysiloxane has both the characteristics of PU and polysiloxane and therefore not only has excellent resistance to both different climates and water but also excellent flexibility, adhesive strength, and a low surface energy, as well as good moisture management and air permeability [7-9]. Therefore, research work on WPU modified with polysiloxane has received substantial attention. For example, Zong et al. [10] conducted a synthesis of graft and blocked copolymerization-modified polydimethylsiloxane(PDMS-) PU for a silicone block-modified WPU by using the acetone process. With an increase in the PDMS content or molecular weight, the viscosity and particle size of the modified WPU emulsion increase, and the water and solvent resistances of the coating also increase. Fei et al. [11] prepared an internally cross-linked silicone-modified cationic WPU by using hydroxyl-terminated polydimethylsiloxane (HPMS). The solution was then formed into films. It was found that the crystallinity of the films is reduced with an 


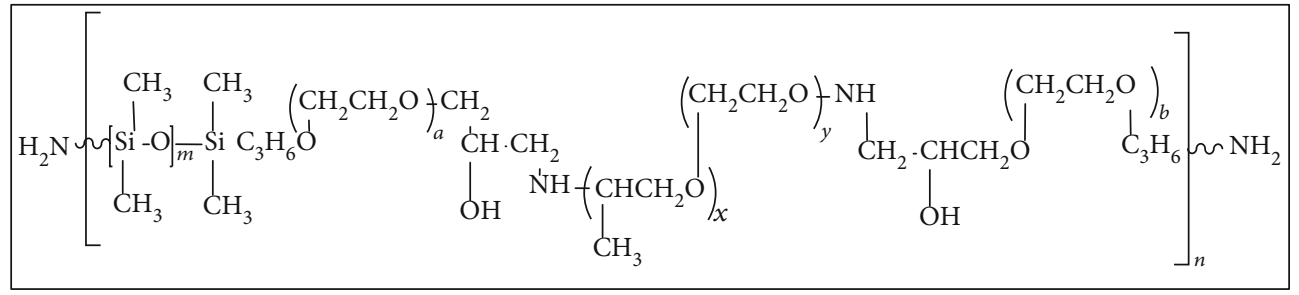

FIgURE 1: Molecular structure of LEPS.

increase in HPMS in the WPU. The modified WPU showed a noncrystalline state, and the microphase separation of the films is increased.

The majority of organosilicon modifiers are hydroxyl and amino silicone oils and silane coupling agents. However, studies on the use of a linear polyether-blocked amino silicone (LEPS) to synthesize a new structure of WPU have been rarely carried out [12].

At present, research work on WPU modified with polysiloxane has mainly focused on anion WPU [13-15], while there are some although relatively few reports on cationic WPU [16]. Cationic WPU is positively electroactive and insensitive to hard water. Cationic WPU is easy to use with a certain bactericidal effect, especially on electroactive substrates with a negative charge, such as textiles, leather, and paper. However, these are also flexible substrates which require a soft WPU film. For this reason, an NCOterminated cationic WPU is synthesized in this study by using polytetramethylene ether glycol (PTMEG) as the soft segment, isophorone diisocyanate (IPDI) to form the hard segment, and BDO and n-methyldiethanolamine (MDEA) as the chain extenders. Moreover, a modified cationic WPU emulsion and its films are created through a reaction between the WPU prepolymer and linear polyether-blocked amino silicone (LEPS), which is an organosilicon compound with a large molecular weight and low ammonia value. The cationic WPU prepolymer is anticipated to have good potential for application in the different fields and industries mentioned earlier. The stability of the modified WPU solution, hardness, adhesion, resistance to water and heat, crystallinity and other properties of the films are examined, and the impacts of adding LEPS on the structure and properties of the WPU emulsion and films are investigated.

\section{Experimental}

2.1. Materials. PTMEG $\left(\mathrm{Mn}=2000 \mathrm{~g} \cdot \mathrm{mol}^{-1}\right)$ was obtained from Hangzhou Sanlong New Material Co., Ltd. (China). IPDI was obtained from Bayer (Germany). MDEA, DBTDL, BDO, acetone, and acetic acid were supplied by Guoyao Chemical Reagent Co., Ltd. (China). LEPS (ammonia value $=0.15 \mathrm{mmol} \cdot \mathrm{g}^{-1}$ and $\mathrm{Mv}=2.5 \times 10^{4} \mathrm{~g} \cdot \mathrm{mo}$ $1^{-1}$ ) was synthesized according to laboratory standards, and its molecular structure is shown in Figure 1 [17].

2.2. Preparation of WPU. To pretreat the raw materials, IPDI, MDEA, and BDO were dried by using a molecular sieve to absorb the water from the reagent. PTMEG was added into
TABLE 1: Compositions of LEPS modified WPU emulsions.

\begin{tabular}{lcccccc}
\hline Sample & $R$ value & $\begin{array}{c}\text { IPDI } \\
(\mathrm{wt} \%)\end{array}$ & $\begin{array}{c}\text { PTMEG } \\
(\mathrm{wt} \%)\end{array}$ & $\begin{array}{c}\text { MDEA } \\
(\mathrm{wt} \%)\end{array}$ & $\begin{array}{c}\text { BDO } \\
(\mathrm{wt} \%)\end{array}$ & $\begin{array}{c}\text { LEPS } \\
(\mathrm{wt} \%)\end{array}$ \\
\hline WPU0 & 1.4 & 41.0 & 48.0 & 6.0 & 5.0 & 0 \\
WPU1 & 1.4 & 39.9 & 45.6 & 6.0 & 5.0 & 3.5 \\
WPU2 & 1.4 & 39.5 & 42.5 & 6.0 & 5.0 & 7.0 \\
WPU3 & 1.4 & 39.1 & 39.4 & 6.0 & 5.0 & 10.5 \\
WPU4 & 1.4 & 38.9 & 36.1 & 6.0 & 5.0 & 14.0 \\
WPU5 & 1.4 & 38.0 & 30.0 & 6.0 & 5.0 & 21.0 \\
\hline
\end{tabular}

a $500 \mathrm{~mL}$ 3-neck flask with a magnetic stirrer, thermometer, and condenser at a temperature of $115^{\circ} \mathrm{C}-120^{\circ} \mathrm{C}$ under vacuum extraction for $60 \mathrm{~min}$ to remove water and contaminants and then cooled to room temperature. During the preparation of the WPU emulsion, the reactor was filled with nitrogen and the PTMEG, IPDI, and DBTDL (0.08 wt $\%$ of the material composition) were placed into a $500 \mathrm{~mL} 3$-neck flask with a magnetic stirrer and a thermometer after pretreatment. The reaction time was $90 \mathrm{~min}$ at a temperature of $80^{\circ} \mathrm{C}$. The $\mathrm{BDO}$ was then added as a chain extender and reacted for $60 \mathrm{~min}$. Then, the reaction temperature was reduced to $55^{\circ} \mathrm{C}$ and the MDEA was subsequently added as the chain extender. The reaction continued for another $90 \mathrm{~min}$ to form the NCO-terminated cationic WPU. Acetone (30 wt $\%$ of the cationic WPU) was added to reduce the viscosity of the reaction. LEPS was then added dropwise into the flask and vigorously stirred to disperse the WPU to form the reaction. The reaction continued for another $60 \mathrm{~min}$ at a temperature of $55^{\circ} \mathrm{C}$. A cross-linked LEPS-modified WPU emulsion that contained 30\% solids was obtained after salification with a certain amount of acetic acid for $20 \mathrm{~min}$ and then emulsified for $50 \mathrm{~min}$ with deionized water under vigorous stirring. The acetone was removed under a reduced pressure by using a vacuum pump. The quantity (in percentage) of the materials used to prepare the modified WPU is shown in Table 1, and the preparation process of the modified WPU is shown in Figure 2.

The WPU emulsions were coated onto a teflon plate, placed at room temperature for $48 \mathrm{~h}$, and then dried for $48 \mathrm{~h}$ at $50^{\circ} \mathrm{C}$ until they reached a constant weight to form the WPU films. The structure and performance of the films were then characterized by using Fourier transform infrared (FTIR) spectrometry, atomic force microscopy (AFM), thermogravimetric analysis (TGA), X-ray diffraction (XRD), and $\mathrm{X}$-ray photoelectron spectroscopy (XPS), as well as by measuring the rigidity of the films, particle size of the WPU 


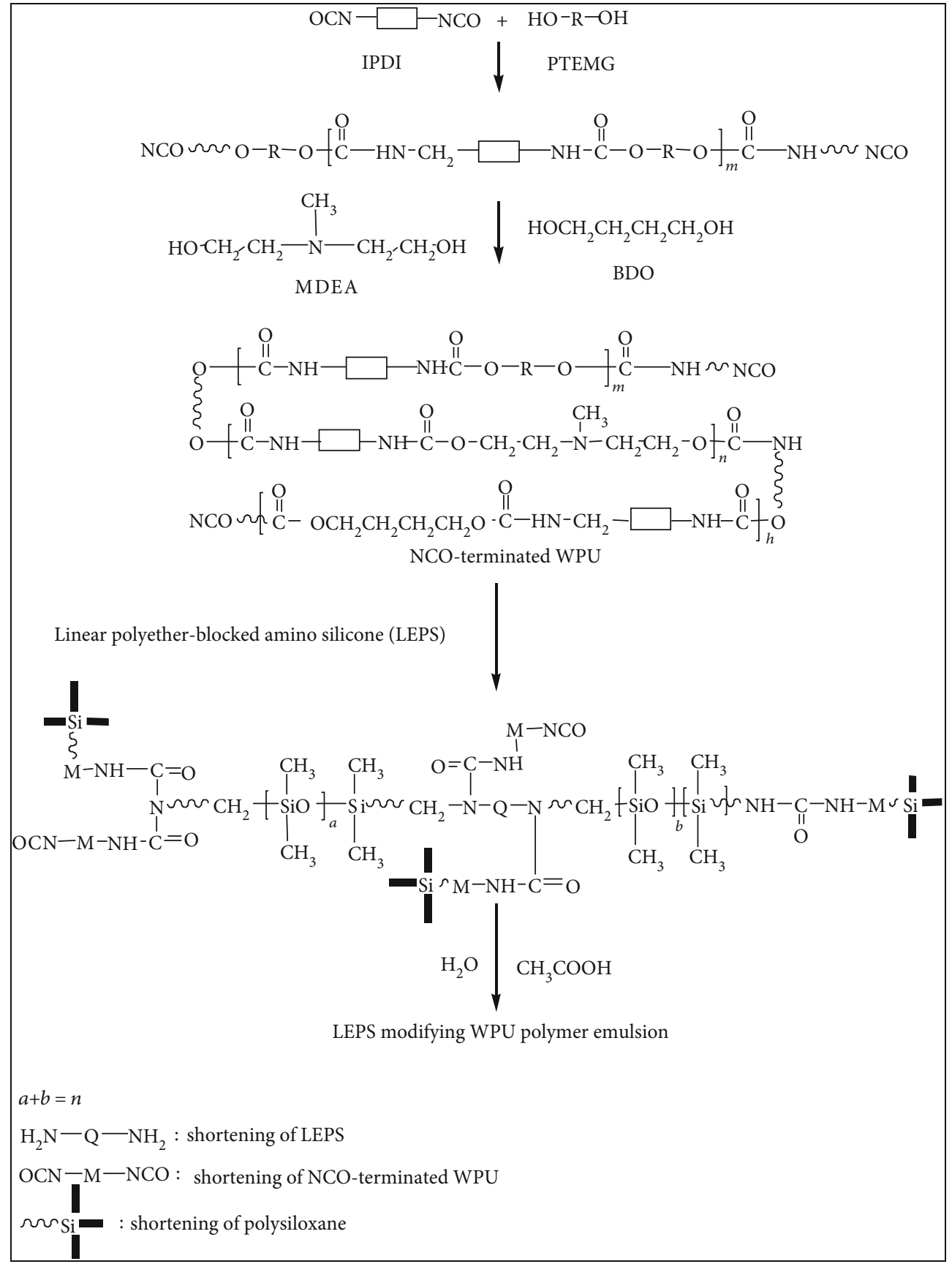

FIGURE 2: Preparation process of modified WPU.

emulsion, and water absorption and water contact angle of the films.

2.3. Testing and Characterization. A Nicolet 5700 Fourier transform infrared spectrometer (Thermo Fisher Scientific, USA) was used to conduct the FTIR spectrometry, and the FTIR spectra of the films were obtained in the transmission mode. The films were grinded together with potassium bromide $(\mathrm{KBr})$ to collect the spectra. The scan range was $500-4000 \mathrm{~cm}^{-1}$, and 100 scans were performed at a frequency of $5 \mathrm{~cm}^{-1}$.

To carry out the surface characterization through AFM, a cover slip was coated with the WPU emulsion at room temperature to prepare the WPU films. Determining the morphology of the WPU films was done by using the tapping mode of a Multimode 3A atomic force microscope (Veeco, USA) with direct contact between the sample surface and the cantilever tip under room temperature.

To carry out the TGA, the WPU films were examined by using a TGA/SDTA851e thermogravimetric analyzer (Mettler Toledo, Switzerland) under a nitrogen gas atmosphere and heated at a rate of $5^{\circ} \mathrm{C} / \mathrm{min}$. The testing was carried out at temperatures that ranged from $50^{\circ} \mathrm{C}$ to $550^{\circ} \mathrm{C}$.

To carry out the XRD testing of the WPU films, a D/Max 2550 PC X-ray diffractometer (Rigaku, Japan) was used with 
copper- (Cu-) K $\alpha$ radiation and a voltage of $40 \mathrm{kV}$, at a scan speed of $0.06^{\circ} / \mathrm{s}$, and scan range of $5^{\circ}-60^{\circ}$.

To carry out the XPS, the WPU films were examined under an XSAM800 photoelectron spectroscope (Kratos, $\mathrm{UK}$ ) and measured with an $\mathrm{Al}$ target. The voltage used to operate the $\mathrm{X}$-ray fluorescence gun was $12 \mathrm{kV}$, current was $15 \mathrm{~mA}$, and the pressure in the analysis chamber was $2 \times 10^{-7} \mathrm{~Pa}$.

The WPU emulsion $(10 \mathrm{ml})$ was added into a centrifuge test tube $(15 \mathrm{ml})$ to determine its stability during centrifugation and centrifuged at $2000 \mathrm{rpm}$ for $20 \mathrm{~min}$.

The rigidity of the WPU films was tested in accordance with the GB/T 6739-2006 (China) Standard Test Method (Paints and varnishes-Determination of film hardness by pencil test), and the adhesion of the WPU films was tested in accordance with the GB/T 9286-1998 (China) Standard Test Method (Paints and varnishes-Cross cut test for films).

The particle size of the WPU emulsion was tested by using a ZetaSizer Nano-ZS laser particle size analyzer (Malvern Instruments, UK).

To determine the water absorption of the films, they were cut into squares with dimensions of $2 \mathrm{~cm} \times 2 \mathrm{~cm}$ and a weight of $M_{1}$. The samples were then dried with a filter paper and weighed $\left(\mathrm{M}_{2}\right)$ after they were immersed into water for $24 \mathrm{~h}$ at room temperature. The water absorption was calculated by using the following equation:

$$
\text { Water absorption }=\frac{M_{2}-M_{1}}{M_{1}} \times 100 \%
$$

The water contact angle of the WPU films was determined by dripping deionized water onto the surface of the WPU films. After $1 \mathrm{~min}$, the contact angle was measured three times for each sample. The average value of the three measurements was used for the analysis. A JC2000C3 contact angle goniometer (Shanghai Zhongcheng Digital Technology Equipment Co., Ltd., China) was used to carry out the contact angle measurements.

\section{Results and Discussion}

3.1. Infrared Spectroscopic Analysis. The infrared spectra of the LEPS, WPU0 (WPU is not modified with LEPS), and WPU4 (WPU modified with 14\% LEPS) samples are shown in Figure 3. It can be observed that the LEPS samples show $\mathrm{Si}-\mathrm{O}-\mathrm{Si}$ stretching vibration peaks at $1022 \mathrm{~cm}^{-1}$ and $1108 \mathrm{~cm}^{-1}$ and $\mathrm{Si}-\mathrm{C}$ characteristic absorption peaks at 803 , 865 and $1240 \mathrm{~cm}^{-1}$. The total content of $-\mathrm{NH},-\mathrm{NH}_{2}$, and $-\mathrm{OH}$ in the LEPS is relatively low, and the mass score is only about $0.6 \%(w / w)$, so there is no corresponding absorption peak in the infrared spectra. It is well known that the stretching vibration ranges of $\mathrm{N}-\mathrm{H}$ and $\mathrm{C}=\mathrm{O}$ in polyurethane are $3300 \mathrm{~cm}^{-1} \sim 3600 \mathrm{~cm}^{-1}$ and $1600 \mathrm{~cm}^{-1} \sim 1900 \mathrm{~cm}^{-1}$, respectively. However, hydrogen bonding is sensitive to the effect of absorption peak, and the absorption peak moves to a low wave number after forming hydrogen bonding [18-20]. It can be observed that there are $\mathrm{N}-\mathrm{H}$ stretching and deformation vibration peaks in the urea group at $3320 \mathrm{~cm}^{-1}$ and $1540 \mathrm{~cm}^{-1}$, respectively, and a $\mathrm{C}=\mathrm{O}$ telescopic vibration peak

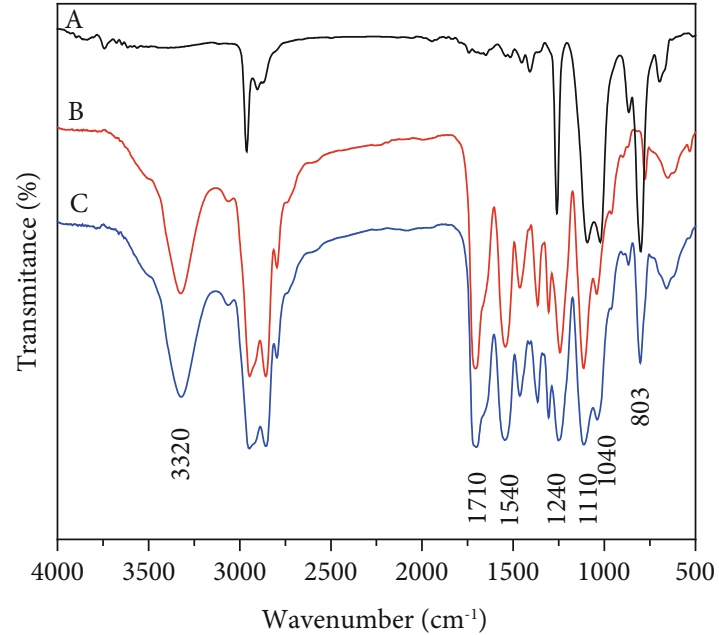

Figure 3: FTIR spectra: (a) LEPS, (b) WPU0, and (c) WPU4.

TABLE 2: Effect of LEPS content on properties of WPU emulsion.

\begin{tabular}{lcccc}
\hline $\begin{array}{l}\text { LEPS } \\
\text { content (\%) }\end{array}$ & Appearance & $\begin{array}{c}\text { Stability during } \\
\text { centrifugation }\end{array}$ & $\begin{array}{c}\text { Average } \\
\text { particle size } \\
(\mathrm{nm})\end{array}$ & PDI \\
\hline 0 & Milky white & Stable & 107.8 & 0.126 \\
3.5 & Milky white & Stable & 125.0 & 0.179 \\
7.0 & Milky white & Stable & 172.1 & 0.381 \\
10.5 & Milky white & Stable & 186.6 & 0.356 \\
14.0 & Milky white & Stable & 203.5 & 0.307 \\
21.0 & Milky white & $\begin{array}{c}\text { Obvious } \\
\text { precipitation }\end{array}$ & 235.6 & 0.165 \\
\hline
\end{tabular}

at $1710 \mathrm{~cm}^{-1}$ of both the WPU0 and WPU4 samples. These vibration peaks indicate the presence of carbamate in both of these samples, and hydrogen bonding are formed in $\mathrm{N}-\mathrm{H}$ and $\mathrm{C}=\mathrm{O}$, respectively. Moreover, the absorption peaks of WPU4 at $1040 \mathrm{~cm}^{-1}$ to $1100 \mathrm{~cm}^{-1}$ are significantly wider and stronger, and this absorption band is similar to that of the LEPS, which is the result of the overlapping of the Si-O-Si and C-O-C stretching vibrations [21]. Meanwhile, the WPU4 curve has a symmetric stretching vibration peak at $1240 \mathrm{~cm}^{-1}$ and a rocking vibration peak at $803 \mathrm{~cm}^{-1}$ due to the composition of $\mathrm{Si}-\mathrm{CH}_{3}$. All of these indicate that LEPS has been successfully incorporated into the WPU chains and the resultant product is consistent with the design structure.

3.2. Emulsion Properties. As the LEPS was introduced into WPU, a polysiloxane segment was incorporated into the WPU emulsion. The molecular weight increased due to cross-linking and chain extension as the small number of amino and imino groups in the LEPS has a certain effect on the emulsion properties. The effect of the LEPS content on the properties of the WPU emulsion is shown in Table 2.

Table 2 indicates that the appearance of the WPU emulsions is milky white in color and WPU emulsions are centrifugally stable when the LEPS content of the WPU is within 
TABLE 3: Rigidity and adhesion performance of WPU films.

\begin{tabular}{lcc}
\hline LEPS content (\%) & Shore hardness (B) & Adhesion (grade) \\
\hline 0 & $3-4$ & 0 \\
3.5 & 4 & 0 \\
7.0 & 4 & 0 \\
10.5 & 5 & 0 \\
14.0 & 5 & 0 \\
\hline
\end{tabular}

$14.0 \mathrm{wt} \%$. When the LEPS content in the WPU is increased to $21 \mathrm{wt} \%$, there is low centrifugal stability of the emulsion. With an increase in the LEPS content in the WPU from 0 to $21 \mathrm{wt} \%$, the particle size of the modified WPU emulsion substantially increases from $107.8 \mathrm{~nm}$ to $235.6 \mathrm{~nm}$, and the particle size dispersion coefficient first increases and then decreases. The introduction of LEPS to WPU increases the molecular weight of the modified WPU due to the chain extension and cross-linking of LEPS and the WPU. The amino and imino groups in the LEPS chain react with -NCO in the WPU to introduce the polysiloxane chain segment which has a high molecular weight. Meanwhile, the hydrophobic polysiloxane segment that is added to the molecular chain causes an increase in the particle size of the WPU emulsion $[22,23]$.

When a small amount of LEPS is introduced, some of the WPU is grafted with the LEPS (modified WPU) while the residual WPU reacts with alcohol or water (untreated WPU). The molecules of the LEPS-modified WPU and untreated WPU are both found in the emulsion, and the particle size of the modified WPU is larger than that of the untreated WPU. Thus, there is an increase in the dispersion of the particle size. However, when the amount of LEPS is increased, there is also the increased likelihood that the WPU has the same degree of copolymerization as LEPS. Therefore, as the amount of LEPS is increased, the probability that the WPU is being modified increases, and the range of the particle size distribution of the WPU emulsion first increases and then decreases. Therefore, in order to ensure the storage stability of the emulsion, the highest amount of LEPS introduced to the WPU was set at $14.0 \mathrm{wt} \%$.

3.3. Rigidity and Adhesion Performance of WPU Films. The effect of the amount of LEPS on the rigidity of the WPU films and their adhesion performance was tested, and the results are shown in Table 3.

Table 3 shows that the shore hardness of the WPU films is reduced from $3-4 \mathrm{~B}$ to $5 \mathrm{~B}$ as the LEPS content in the WPU is increased. The adhesion of the WPU films is not affected by the LEPS content as they all have a 0 grade adhesion, which means excellent adhesion to the substrate.

LEPS is a new type of amino silicone oil with a low ammonia value and large molecular weight, which are the characteristics of polysiloxane [24]. The molecular chain of the LEPS has good flexibility due to the alternating polymerization of the linear-structured polysiloxane and polyether. When subjected to external forces, the external forces can be eliminated through the deformation of its own molecular chain. Moreover, the degree of cross-linking between LEPS

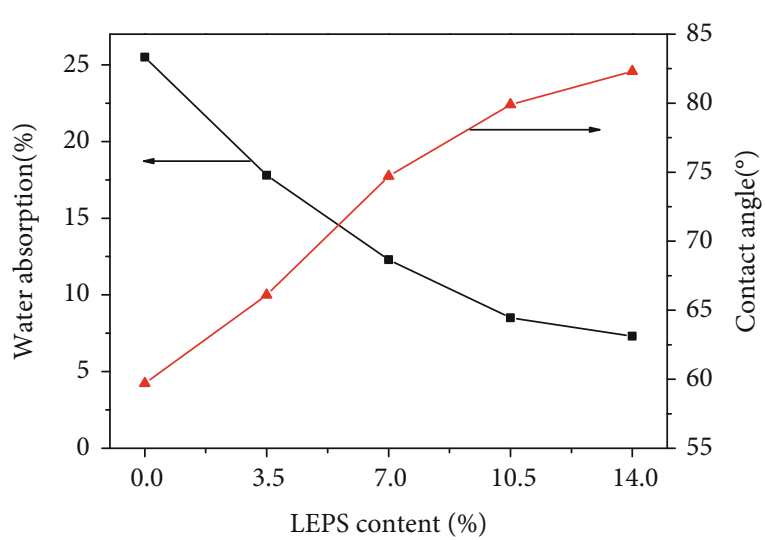

FIGURE 4: Effect of LEPS content on water absorption and contact angle of WPU films.

and WPU is relatively low, and the polysiloxane chain segment can be readily enriched on the surface of the film. Therefore, the LEPS-modified WPU films have good flexibility. In addition, LEPS has a low ammonia value and a small number of hard urea groups produced through the crosslinking of LEPS and PU, which means that they do not contribute much in increasing the shore hardness of the films. Thus, the modified WPU film has more flexibility with a higher LEPS content. Furthermore, the LEPS-modified WPU film is flexible and nonadhesive. As the WPU coating was drying, the polysiloxane segment migrated to the film surface, and there was surface enrichment of the film as the compatibility between polysiloxane and PU is low and the degree of cross-linking between the two is also low. On the other hand, the polysiloxane chain segment on the adhesive surface of the coating is shorter. Therefore, the adhesion of WPU to the substrate is not affected by the modification with LEPS.

3.4. Water Absorption and Contact Angle Analysis. The results of the water absorption test and contact angle measurements of the WPU films are shown in Figure 4.

It can be seen that with an increase in the content of LEPS in the WPU from 0 to $14 \mathrm{wt} \%$, the water absorption of the film is substantially reduced from $25.5 \%$ to $7.5 \%$ while the contact angle is significantly increased from $59.7^{\circ}$ to $82.3^{\circ}$. Modification with LEPS has two effects on the resistance of WPU to water. First, the compatibility of the polysiloxane chain segment and PU in the modified WPU is low, which enhances the microphase separation of the film. Furthermore, this leads to the ease of diffusion of the water molecules in the film and increases the permeability of the film. Then, after modification of the WPU with LEPS, the hydrophobic polysiloxane segment was introduced to the WPU which, to some extent, inhibited the diffusion of the water molecules in the WPU film and reduced the adsorption of the film in water. Therefore, the water resistance of the LEPS-modified WPU films is improved. Figure 4 shows that the permeability of the WPU film is reduced after modification with LEPS, which indicates that the introduction of a polysiloxane chain segment has a much stronger effect on increasing the water resistance of the film than the decrease caused by an 


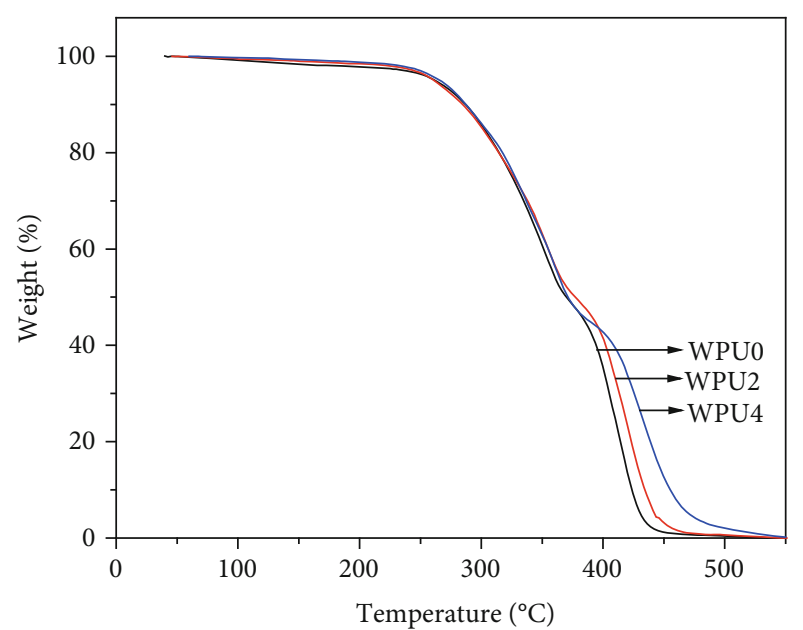

(a)

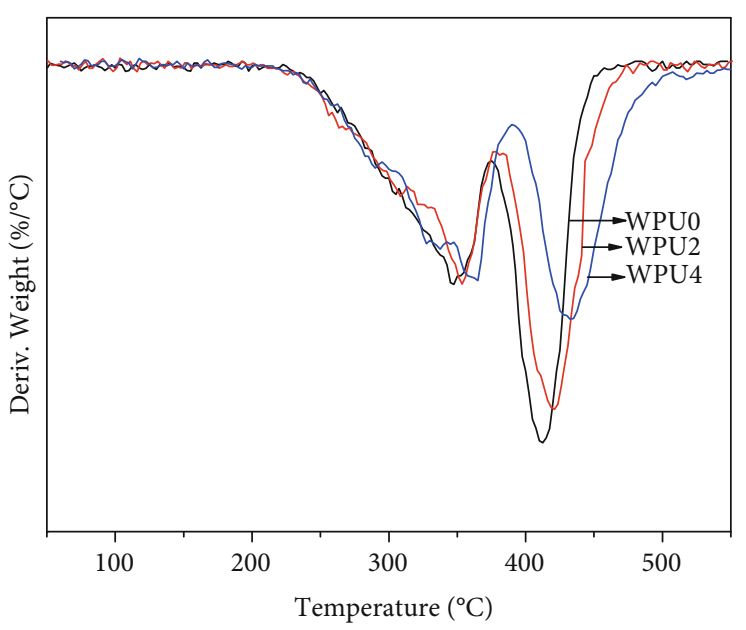

(b)

FIgURe 5: Thermal performance of WPU films: (a) TGA curve of LEPS-modified WPUs and (b) DTG curve of LEPS-modified WPUs.

TABLE 4: TG results of WPU films

\begin{tabular}{lccccc}
\hline Sample & $T_{5}\left({ }^{\circ} \mathrm{C}\right)$ & $T_{50}\left({ }^{\circ} \mathrm{C}\right)$ & $T_{90}\left({ }^{\circ} \mathrm{C}\right)$ & $T_{1 \max }\left({ }^{\circ} \mathrm{C}\right)$ & $T_{2 \max }\left({ }^{\circ} \mathrm{C}\right)$ \\
\hline WPU0 & 262 & 370 & 422 & 329 & 391 \\
WPU2 & 260 & 378 & 434 & 335 & 400 \\
WPU4 & 265 & 373 & 455 & 346 & 412 \\
\hline
\end{tabular}

Note: $T_{5}, T_{50}, T_{90}, T_{1 \max }$, and $T_{2 \max }$ represent the temperature that corresponds to a $5 \%, 50 \%$, and $90 \%$ weight loss of the WPU films and the maximum weight loss in the first and second stages, respectively.

enhanced microphase separation. As mentioned earlier, the polysiloxane chain segment migrated to the surface of the film during film formation, and there was surface enrichment of the film, which reduced the surface tension of the film and increased the water contact angle.

3.5. Thermal Performance of WPU Films. The TGA and DTG curves of the LEPS-modified WPU are shown in Figures 5(a) and 5(b), respectively, and the weight loss results of the samples due to thermal degradation are shown in Table 4.

Figure 4 and Table 4 show that the introduction of LEPS to WPU has no negative effect on the initial weight loss temperature or $5 \%$ weight loss temperature $\left(T_{5}\right)$ and $50 \%$ weight loss temperature $\left(T_{50}\right)$ of the WPU films. Moreover, when compared to the $90 \%$ weight loss temperature $\left(T_{90}\right)$ of WPU0, there is an increase of the $T_{90}$ of WPU2 (WPU modified with $7 \%$ LEPS) and WPU4 to $12^{\circ} \mathrm{C}$ and $33^{\circ} \mathrm{C}$, respectively. In addition, there are two thermal degradation stages of WPU0: the first stage in which the maximum weight loss temperature is $329^{\circ} \mathrm{C}\left(T_{1 \max }\right)$ which is the maximum thermal decomposition temperature of the hard segment in the WPU. The second stage is when the maximum weight loss temperature is $391^{\circ} \mathrm{C}\left(T_{2 \max }\right)$ which is the maximum thermal decomposition temperature of the soft segment in the WPU, and this is consistent with the thermal decomposition characteristics of the typical soft and hard segments of PU $[25,26]$. When $T_{1 \max }$ and $T_{2 \max }$ of WPU0 are compared, there is an increase in the $T_{1 \max }$ of WPU2 and WPU4 to $6^{\circ} \mathrm{C}$ and $17^{\circ} \mathrm{C}$ and an increase in the $T_{2 \max }$ of WPU2 and WPU4 to $9^{\circ} \mathrm{C}$ and $21^{\circ} \mathrm{C}$, respectively. The thermal performance analysis shows that WPU that has been modified with LEPS has significantly improved heat resistance.

3.6. WPU Film Morphology Analysis. The morphology structure of the WPU films observed through AFM with different amounts of LEPS is shown in Figure 6.

Figure 6(a) shows that there is an obvious microphase separation between the convex-shaped IPDI hard segment and the concave-shaped soft segment of the surface of the WPU0 film. The introduction of polysiloxane chains that have low compatibility with PU into WPU by adding LEPS should have facilitated the formation of the microphase separation, while the surface of the WPU2 and WPU4 films is smooth, and no obvious microphase separation can be observed. Possible explanations are the large difference between the solubility parameters of polysiloxane and PU [27] and that the compatibility of polysiloxane and PU is low. Moreover, the low cross-linking density of LEPS and PU promotes the migration of polysiloxane to the surface of the WPU film and enrichment of the surface, and the result is that the surface of the WPU film is completely covered by the LEPS segment. Therefore, the microphase separation of the hard and soft segments of the WPU is not observed. The XPS result for the WPU films below also provides specific evidence.

3.7. Elementary Analysis of Surface of WPU Film. The XPS spectrum of the WPU films before and after modification with LEPS is shown in Figure 7, and the element composition is provided in Table 5. It can be seen in Figure 7 that the untreated WPU film shows element peaks, including carbon $(\mathrm{C})$, oxygen $(\mathrm{O})$, and nitrogen $(\mathrm{N})$ peaks. As for the spectrum of the WPU2 sample, there are the characteristic peaks of Si2p and Si2s with a binding energy of $102 \mathrm{eV}$ and $155 \mathrm{eV}$, respectively, aside from the $\mathrm{C}, \mathrm{O}$, and $\mathrm{N}$ peaks. The spectrum of the WPU4 sample shows the characteristic peaks of Si2p and Si2s with a binding energy of $102 \mathrm{eV}$ and $155 \mathrm{eV}$, 


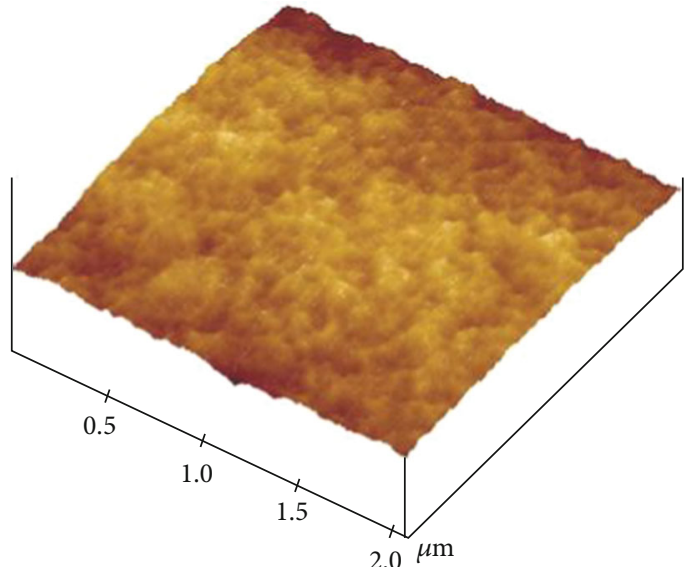

(a)

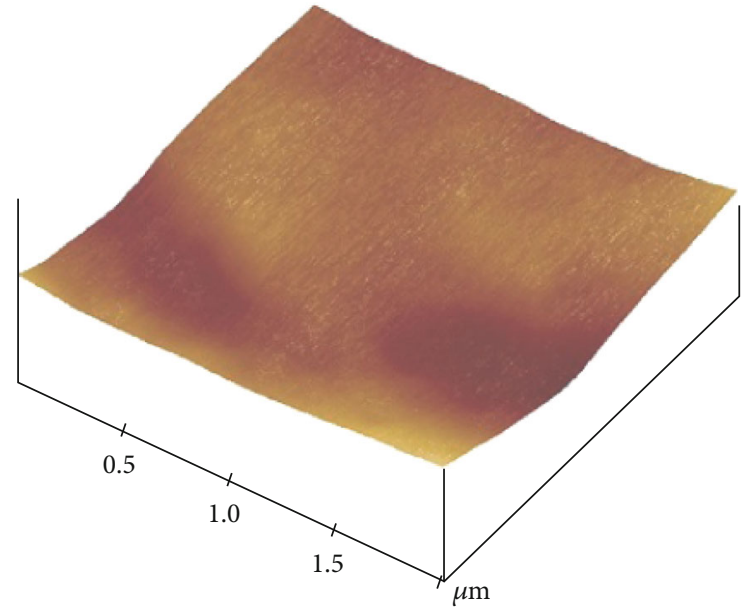

(b)

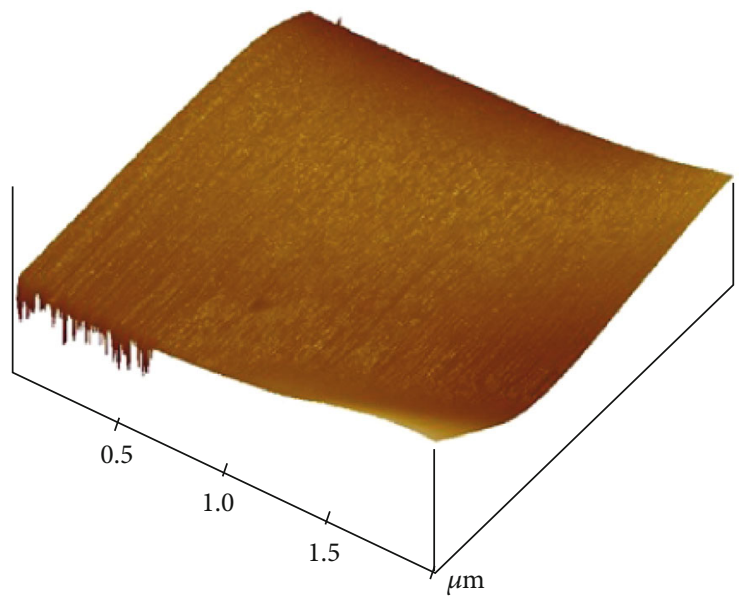

(c)

Figure 6: AFM images of (a) WPU0, (b) WPU2, and (c) WPU4.

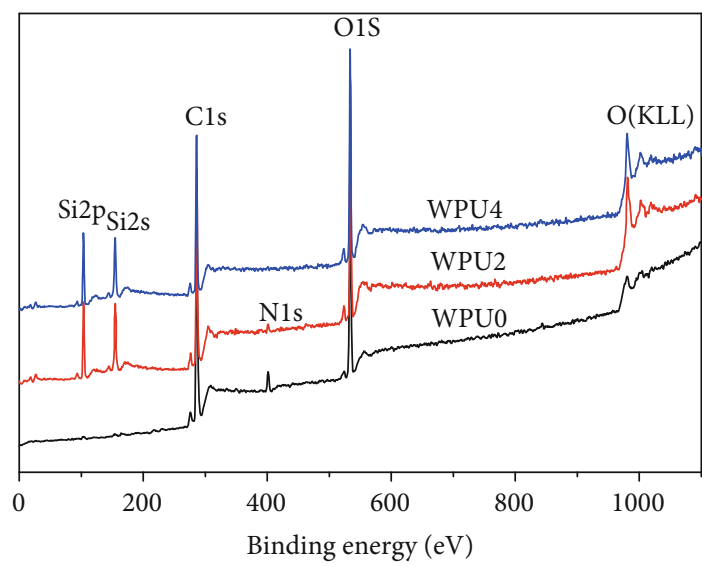

FIGURE 7: XPS spectrum of LEPS-modified WPU films.

respectively, as well as $\mathrm{C}$ and $\mathrm{O}$ peaks, but no $\mathrm{N}$ peak can be detected. The silicon $(\mathrm{Si})$ percentage of the LEPS tested by using XPS is $22.83 \%$. Therefore, the Si percentage of the WPU2 and WPU4 films is theoretically $2.28 \%$ and $4.56 \%$, respectively. However, it can be seen in Table 5 that the $\mathrm{Si}$
TABLE 5: Element composition of LEPS-modified WPU films.

\begin{tabular}{lcccc}
\hline \multirow{2}{*}{ Sample } & \multicolumn{4}{c}{ Element (\%) } \\
& $\mathrm{C}$ & $\mathrm{O}$ & $\mathrm{N}$ & $\mathrm{Si}$ \\
\hline WPU0 & 70.05 & 24.76 & 5.19 & 0 \\
WPU2 & 55.53 & 25.79 & 1.51 & 17.17 \\
WPU4 & 52.08 & 27.52 & 0.00 & 20.40 \\
\hline
\end{tabular}

percentage of the WPU2 and WPU4 samples is actually $17.17 \%$ and $20.40 \%$, respectively. This means that there is an increase in the polysiloxane segments on the surface of the LEPS-modified WPU films, which in turn means that there is an increase of $\mathrm{Si}$ and $\mathrm{O}$ on the surface of the films while the $\mathrm{C}$ and $\mathrm{N}$ contents are obviously reduced compared to the surface of the untreated WPU film. When the percentage of LEPS in the WPU is $14.0 \mathrm{wt} \%$, the percentage of Si is close to that of the LEPS, which indicates that the polysiloxane chain segment on the surface of the WPU film almost completely covers the PU chain segment, which results in the removal of $\mathrm{N}$. 


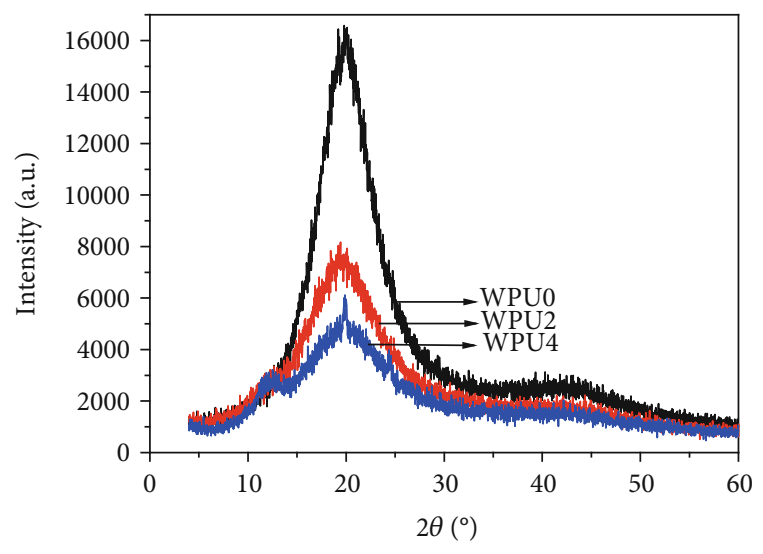

FIGURE 8: XRD patterns of LEPS-modified WPU films.

3.8. Crystallization Analysis. The XRD patterns of the WPU films before and after modification with LEPS are shown in Figure 8.

It can be seen in Figure 8 that regardless whether there is modification, the XRD patterns all show dispersion diffraction peaks, and the diffraction peaks are almost all found at the diffraction angle of $20^{\circ}$. After WPU is modified with LEPS, it can be observed in Figure 8 that the LEPS reduces the XRD peak intensity of the WPU films while the dispersion of the diffraction peak becomes more obvious which is due to the numerous amorphous microcrystalline and subcrystalline states in the system [28, 29].

The results in this study show that PTMG and IPDI cannot form a crystalline continuous phase and only amorphous PU can be obtained. Nevertheless, modification with LEPS affects the crystallinity of WPU, which can be summarized as follows: first, the steric hindrance of the polysiloxane and the formed cross-linked structure after modification reduce the ordered arrangement of the PU chains and their crystallinity. Secondly, the urea group produced after modification with LEPS enhances the function of the hydrogen bonds, which is conducive to the formation of an ordered structure, thus enhancing the molecular chain alignment and improving the crystallinity of the modified WPU. In general, modification with LEPS reduces the crystallization performance of WPU and compatibility between polysiloxane and PU, which promote the formation of the microphase separation. The effects increase and become even more obvious when more LEPS is added to the WPU.

\section{Conclusion}

A cationic WPU modified with flexible polysiloxane is synthesized by using PTMEG, IPDI, BDO, MDEA, and LEPS as the main material. With higher percentages of LEPS in the WPU, the particle size of the modified WPU emulsion is significantly increased, while the centrifugal stability of the emulsion is reduced. Moreover, the WPU films are more thermally stable at high temperatures and the LEPS-modified WPU films are less permeable to water, while the crystallization of the WPU films is reduced. Obviously, there are more polysiloxane segments on the surface of the LEPS-modified
WPU films. Furthermore, the LEPS-modified WPU films are flexible and nonadhesive which is determined through touch. Modification with LEPS has no negative effect on the adhesion between the WPU film and substrate. Moreover, an LEPS content of $14.0 \mathrm{wt} \%$ in the WPU provides the WPU emulsion and film with an overall optimum performance. Therefore, WPU that is modified with LEPS has promising applications in textile and leather and as an alternative flexible substrate coating.

\section{Data Availability}

The data used to support the findings of this study are available from the corresponding author upon request.

\section{Conflicts of Interest}

No potential conflict of interest was reported by the authors.

\section{Acknowledgments}

The authors acknowledge support from the Shaanxi Province Key Research and Development Program project of China (Grant No.: 2018GY-098) and Science and Technology Project of Shaanxi, China (Grant No.: 2017JQ2006), and the Textile Science and Engineering Discipline Construction Project and Green Printing and Dyeing Processing Innovation Team Support Project of the Xi'an Polytechnic University (TD-13).

\section{References}

[1] X. Zhou, Y. Li, C. Fang et al., "Recent advances in synthesis of waterborne polyurethane and their application in water-based ink: a review," Journal of Materials Science \& Technology, vol. 31, no. 7, pp. 708-722, 2015.

[2] A. Noreen, K. M. Zia, M. Zuber, S. Tabasum, and M. J. Saif, "Recent trends in environmentally friendly water-borne polyurethane coatings: a review," Korean Journal of Chemical Engineering, vol. 33, no. 2, pp. 388-400, 2016.

[3] M. B. Kale, Z. Luo, X. Zhang et al., "Waterborne polyurethane/graphene oxide-silica nanocomposites with improved mechanical and thermal properties for leather coatings using screen printing," Polymer, vol. 170, pp. 43-53, 2019.

[4] K. T. Kim, T. D. Dao, H. M. Jeong, R. V. Anjanapura, and T. M. Aminabhavi, "Graphene coated with alumina and its utilization as a thermal conductivity enhancer for alumina sphere/thermoplastic polyurethane composite," Materials Chemistry and Physics, vol. 153, pp. 291-300, 2015.

[5] D. A. Nguyen, A. V. Raghu, J. T. Choi, and H. M. Jeong, "Properties of thermoplastic polyurethane/functionalised graphene sheet nanocomposites prepared by the in situ polymerisation method," Polymers and Polymer Composites, vol. 18, no. 7, pp. 351-358, 2010.

[6] A. Papagiannopoulos, C. M. Fernyhough, T. A. Waigh, and A. Radulescu, "Scattering study of the structure of polystyrene sulfonate comb polyelectrolytes in solution," Macromolecular Chemistry and Physics, vol. 209, no. 24, pp. 2475-2486, 2008.

[7] Q. Li, L. Guo, T. Qiu, W. Xiao, D. du, and X. Li, "Synthesis of waterborne polyurethane containing alkoxysilane side groups 
and the properties of the hybrid coating films," Applied Surface Science, vol. 377, pp. 66-74, 2016.

[8] P. Majumdar and D. C. Webster, "Influence of solvent composition and degree of reaction on the formation of surface microtopography in a thermoset siloxane-urethane system," Polymer, vol. 47, no. 11, pp. 4172-4181, 2006.

[9] K. Madhavan and B. S. R. Reddy, "Synthesis and characterization of poly (dimethylsiloxane-urethane) elastomers: effect of hard segments of polyurethane on morphological and mechanical properties," Journal of Polymer Science Part A: Polymer Chemistry, vol. 44, no. 9, pp. 2980-2989, 2006.

[10] J. Zong, Q. Zhang, H. Sun, Y. Yu, S. Wang, and Y. Liu, "Characterization of polydimethylsiloxane-polyurethanes synthesized by graft or block copolymerizations," Polymer Bulletin, vol. 65 , no. 5, pp. 477-493, 2010.

[11] G. Fei, Y. Shen, H. Wang, and Y. Shen, "Effects of polydimethylsiloxane concentration on properties of polyurethane/polydimethylsiloxane hybrid dispersions," Journal of Applied Polymer Science, vol. 102, no. 6, pp. 5538-5544, 2006.

[12] Y. Yu and J. Wang, "Synthesis and properties of block and graft waterborne polyurethane modified with $\alpha, \omega$ bis(3-(1-methoxy-2-hydroxypropoxy)propyl)polydimethylsiloxane and $\alpha$-N,N-dihydroxyethylaminopropyl- $\omega$-butylpolydimethylsiloxane," Polymer Engineering and Science, vol. 54, no. 4, pp. 805-811, 2014.

[13] C. Zhang, X. Zhang, J. Dai, and C. Bai, "Synthesis and properties of PDMS modified waterborne polyurethane-acrylic hybrid emulsion by solvent-free method," Progress in Organic Coatings, vol. 63, no. 2, pp. 238-244, 2008.

[14] S. K. Lee and B. K. Kim, "High solid and high stability waterborne polyurethanes via ionic groups in soft segments and chain termini," Journal of Colloid and Interface Science, vol. 336, no. 1, pp. 208-214, 2009.

[15] Y. Huang, H. Yu, and C. Xiao, "Effects of $\mathrm{Ca}^{2+}$ crosslinking on structure and properties of waterborne polyurethanecarboxymethylated guar gum films," Carbohydrate Polymers, vol. 66, no. 4, pp. 500-513, 2006.

[16] D. Liu, G. M. Wu, and Z. W. Kong, "Preparation and characterization of a polydimethylsiloxane-modified, epoxy-resinbased polyol dispersion and its crosslinked films," Journal of Applied Polymer Science, vol. 134, no. 1, article 44342, 2017.

[17] C. Xu, L. OuYang, H. Liu et al., "Synthesis of blocking polyether silicone oil and silicone blocking waterborne polyurethane and application to cashmere knitted fabric finishing," Textile Research Journal, vol. 85, no. 19, pp. 2040-2050, 2015.

[18] A. V. Raghu, G. S. Gadaginamath, and T. M. Aminabhavi, "Synthesis and characterization of novel polyurethanes based on 1,3-bis(hydroxymethyl) benzimidazolin-2-one and 1,3bis(hydroxymethyl) benzimidazolin-2-thione hard segments," Journal of Applied Polymer Science, vol. 98, no. 5, pp. 22362244, 2005.

[19] A. V. Raghu, G. S. Gadaginamath, M. Priya, P. Seema, H. M. Jeong, and T. M. Aminabhavi, "Synthesis and characterization of novel polyurethanes based onN1,N4-bis[(4-hydroxyphenyl)methylene]succinohydrazide hard segment," Journal of Applied Polymer Science, vol. 110, no. 4, pp. 2315-2320, 2008.

[20] A. V. Raghu, Y. R. Lee, H. M. Jeong, and C. M. Shin, "Preparation and physical properties of waterborne polyurethane/functionalized graphene sheet nanocomposites," Macromolecular Chemistry and Physics, vol. 209, no. 24, pp. 2487-2493, 2008.
[21] H. Chen, Q. Fan, D. Chen, and X. Yu, "Synthesis and properties of polyurethane modified with an aminoethylaminopropylsubstituted polydimethylsiloxane.II. Waterborne polyurethanes," Journal of Applied Polymer Science, vol. 79, no. 2, pp. 295-301, 2001.

[22] C. Xu, L. OuYang, Z. Cai, Y. Ren, S. Lu, and W. Shi, "Effects of polyaminosiloxane on the structure and properties of modified waterborne polyurethane," Journal of Applied Polymer Science, vol. 136, no. 12, p. 47226, 2019.

[23] H. Zhou, H. Wang, X. Tian, K. Zheng, and Q. Cheng, "Effect of 3-Aminopropyltriethoxysilane on polycarbonate based waterborne polyurethane transparent coatings," Progress in Organic Coatings, vol. 77, no. 6, pp. 1073-1078, 2014.

[24] C. Xu, M. Wu, Y. Ren, J. Xing, Z. Cai, and L. Ouyang, "Preparation and properties of linear polyether-blocked amino silicone," Journal of Textile Research, vol. 40, pp. 89-94, 2019.

[25] A. Vuillequez, J. Moreau, M. R. Garda, B. Youssef, and J. M. Saiter, "Polyurethane methacrylate/silicone interpenetrating polymer networks synthesis, thermal and mechanical properties," Journal of Polymer Research, vol. 15, no. 2, pp. 89-96, 2008.

[26] A. Rekondo, L. Irusta, and M. J. Fernández-Berridi, "Characterization of silanized poly(ether-urethane) hybrid systems using thermogravimetric analysis (TG)," Journal of Thermal Analysis and Calorimetry, vol. 101, no. 1, pp. 331-337, 2010.

[27] S. Xu, L. Xie, X. Yu, Y. Xiong, and H. Tang, "Synthesis and characterization of phenyl polysiloxane modified polyurea/polyurethanes," Journal of Polymer Science Part A: Polymer Chemistry, vol. 53, no. 15, pp. 1794-1805, 2015.

[28] S. Mondal and J. L. Hu, "Structural characterization and mass transfer properties of nonporous segmented polyurethane membrane: influence of hydrophilic and carboxylic group," Journal of Membrane Science, vol. 274, no. 1-2, pp. 219-226, 2006.

[29] W. K. Lee, "Synthesis of polyester/poly(dimethylsiloxane)/polyester triblock copolymers and their rearrangement under water: rearrangement of PDMS-containing copolymers," Composite Interfaces, vol. 13, no. 2-3, pp. 159-171, 2006. 